\title{
Wigner function description of a qubit-oscillator system
}

\author{
James Allen and A.M. Zagoskin \\ Loughborough University, Loughborough, Leics LE11 3TU, UK \\ E-mail: A.Zagoskin@eboro.ac.uk
}

Received October 31, 2012 \begin{abstract}
near oscillator, and investigate their usefulness in the quantum-classical transition regime.
PACS: 03.67.-a Quantum information;

03.67.Lx Quantum computation architectures and implementations;

05.30.-d Quantum statistical mechanics.
\end{abstract}

We derive the Bloch-type equations of motion for the Wigner function of a qubit nonlinearly coupled to a li-

Keywords: qubit, oscillator, coherent state, Wigner function, Bloch equations, cat states, collapse and revival.

Phase-space description of quantum systems is especially advantageous for the purpose of investigation of quantum-classical transition regime and in quantum optics, due to the quasiprobability properties of the Wigner function and to its natural representation in the basis of coherent states $[1,2]$. It also provided a convenient tool for restoring a full quantum state from a finite set of measurements (quantum tomography), both in quantum optics and in quantum computing $[3,4]$, in particular in application to superconducting qubits [5,6]. Generalizations of the Wigner function were proposed to describe discrete degrees of freedom (see, e.g., [7,8]).

Here we apply the Wigner-function based approach to a simple model: a two level system coupled to a linear oscillator. This system quite accurately describes such objects as qubits coupled to control circuits [9], and therefore has important applications. Our goal is to derive equations for the Wigner function of the system, which could serve as a convenient starting point for both numerical and analytical treatment and to provide a straightforward method of finding correlators and spectral densities of relevant observables.

We limit our consideration of nonlinear qubit-oscillator coupling to the quadratic terms. Thus, the Hamiltonian is given by

$$
\begin{gathered}
H=H_{0}+H_{I} \\
H_{0}=\frac{1}{2} \hbar \Omega \sigma_{z}+\hbar \omega a^{\dagger} a \\
H_{I}=g_{1} \sigma_{x}\left(a+a^{\dagger}\right)+g_{2} \sigma_{z}\left(a+a^{\dagger}\right)+g_{3} \sigma_{z}\left(a+a^{\dagger}\right)^{2} .
\end{gathered}
$$

The Liouville-von Neumann equation of motion for the density matrix,

$$
i \hbar \frac{\partial}{\partial t} \rho=\left[H_{0}+H_{I}, \rho\right]
$$

yields the equations for the components:

$$
\begin{gathered}
i \hbar \frac{\partial}{\partial t} \rho_{11}=\hbar \omega a^{\dagger} a \rho_{11}-\hbar \omega \rho_{11} a^{\dagger} a+g_{1}\left(a+a^{\dagger}\right) \rho_{21}-g_{1} \rho_{12}\left(a+a^{\dagger}\right)+g_{2}\left(a+a^{\dagger}\right) \rho_{11}-g_{2} \rho_{11}\left(a+a^{\dagger}\right)+ \\
+g_{3}\left(a^{2}+a a^{\dagger}+a^{\dagger} a+a^{\dagger 2}\right) \rho_{11}-g_{3} \rho_{11}\left(a^{2}+a a^{\dagger}+a^{\dagger} a+a^{\dagger 2}\right) ; \\
i \hbar \frac{\partial}{\partial t} \rho_{12}=\hbar \Omega \rho_{12}+\hbar \omega a^{\dagger} a \rho_{12}-\hbar \omega \rho_{12} a^{\dagger} a+g_{1}\left(a+a^{\dagger}\right) \rho_{22}-g_{1} \rho_{11}\left(a+a^{\dagger}\right)+g_{2}\left(a+a^{\dagger}\right) \rho_{12}+g_{2} \rho_{12}\left(a+a^{\dagger}\right)+ \\
+g_{3}\left(a^{2}+a a^{\dagger}+a^{\dagger} a+a^{\dagger 2}\right) \rho_{12}+g_{3} \rho_{12}\left(a^{2}+a a^{\dagger}+a^{\dagger} a+a^{\dagger 2}\right) ; \\
i \hbar \frac{\partial}{\partial t} \rho_{21}=-\hbar \Omega \rho_{21}+\hbar \omega a^{\dagger} a \rho_{21}-\hbar \omega \rho_{21} a^{\dagger} a+g_{1}\left(a+a^{\dagger}\right) \rho_{11}-g_{1} \rho_{22}\left(a+a^{\dagger}\right)-g_{2}\left(a+a^{\dagger}\right) \rho_{21}-g_{2} \rho_{21}\left(a+a^{\dagger}\right)- \\
-g_{3}\left(a^{2}+a a^{\dagger}+a^{\dagger} a+a^{\dagger 2}\right) \rho_{21}-g_{3} \rho_{21}\left(a^{2}+a a^{\dagger}+a^{\dagger} a+a^{\dagger 2}\right) ;
\end{gathered}
$$




$$
\begin{gathered}
i \hbar \frac{\partial}{\partial t} \rho_{22}=\hbar \omega a^{\dagger} a \rho_{22}-\hbar \omega \rho_{22} a^{\dagger} a+g_{1}\left(a+a^{\dagger}\right) \rho_{12}-g_{1} \rho_{21}\left(a+a^{\dagger}\right)-g_{2}\left(a+a^{\dagger}\right) \rho_{22}+g_{2} \rho_{22}\left(a+a^{\dagger}\right)- \\
-g_{3}\left(a^{2}+a a^{\dagger}+a^{\dagger} a+a^{\dagger 2}\right) \rho_{22}+g_{3} \rho_{22}\left(a^{2}+a a^{\dagger}+a^{\dagger} a+a^{\dagger 2}\right) .
\end{gathered}
$$

Each of the components $\rho_{i j}$ is an infinitely dimensional matrix in the Fock space of the oscillator. The transformation to the phase space variables $\alpha, \alpha^{*}$ produces a set of differential equations for the components $W_{i j}\left(\alpha, \alpha^{*}\right)$ according to the rule [1]

$$
a^{\dagger} \rho=\left(\alpha^{*}-\frac{1}{2} \frac{\partial}{\partial \alpha}\right) W ; \quad a \rho=\left(\alpha+\frac{1}{2} \frac{\partial}{\partial \alpha^{*}}\right) W ; \quad \rho a^{\dagger}=\left(\alpha^{*}+\frac{1}{2} \frac{\partial}{\partial \alpha}\right) W ; \quad \rho a=\left(\alpha-\frac{1}{2} \frac{\partial}{\partial \alpha^{*}}\right) W
$$

This yields

$$
\begin{aligned}
& i \hbar \frac{\partial}{\partial t} W_{11}=\hbar \omega\left(\alpha^{*} \frac{\partial}{\partial \alpha^{*}}-\frac{\partial}{\partial \alpha} \alpha\right) W_{11}+g_{1}\left(\alpha+\alpha^{*}+\frac{1}{2} \frac{\partial}{\partial \alpha^{*}}-\frac{1}{2} \frac{\partial}{\partial \alpha}\right) W_{21}- \\
& -g_{1}\left(\alpha+\alpha^{*}-\frac{1}{2} \frac{\partial}{\partial \alpha^{*}}+\frac{1}{2} \frac{\partial}{\partial \alpha}\right) W_{12}+g_{2}\left(\frac{\partial}{\partial \alpha^{*}}-\frac{\partial}{\partial \alpha}\right) W_{11}+g_{3}\left(2 \alpha \frac{\partial}{\partial \alpha^{*}}-\alpha \frac{\partial}{\partial \alpha}+\frac{\partial}{\partial \alpha^{*}} \alpha^{*}+\alpha^{*} \frac{\partial}{\partial \alpha^{*}}-\frac{\partial}{\partial \alpha} \alpha-2 \alpha^{*} \frac{\partial}{\partial \alpha}\right) W_{11} \\
& i \hbar \frac{\partial}{\partial t} W_{12}=\hbar \Omega W_{12}+\hbar \omega\left(\alpha^{*} \frac{\partial}{\partial \alpha^{*}}-\frac{\partial}{\partial \alpha} \alpha\right) W_{12}+g_{1}\left(\alpha+\alpha^{*}+\frac{1}{2} \frac{\partial}{\partial \alpha^{*}}-\frac{1}{2} \frac{\partial}{\partial \alpha}\right) W_{22}- \\
& -g_{1}\left(\alpha+\alpha^{*}-\frac{1}{2} \frac{\partial}{\partial \alpha^{*}}+\frac{1}{2} \frac{\partial}{\partial \alpha}\right) W_{11}+g_{2}\left(2 \alpha+2 \alpha^{*}\right) W_{12}+g_{3}\left(2 \alpha^{2}+\frac{1}{2} \frac{\partial^{2}}{\partial \alpha^{* 2}}+4 \alpha \alpha^{*}+2 \alpha^{* 2}-\frac{\partial^{2}}{\partial \alpha^{*} \partial \alpha}+\frac{1}{2} \frac{\partial^{2}}{\partial \alpha^{2}}\right) W_{12} \\
& i \hbar \frac{\partial}{\partial t} W_{21}=\hbar \Omega W_{21}+\hbar \omega\left(\alpha^{*} \frac{\partial}{\partial \alpha^{*}}-\frac{\partial}{\partial \alpha} \alpha\right) W_{12}+g_{1}\left(\alpha+\alpha^{*}+\frac{1}{2} \frac{\partial}{\partial \alpha^{*}}-\frac{1}{2} \frac{\partial}{\partial \alpha}\right) W_{11}- \\
& -g_{1}\left(\alpha+\alpha^{*}-\frac{1}{2} \frac{\partial}{\partial \alpha^{*}}+\frac{1}{2} \frac{\partial}{\partial \alpha}\right) W_{22}-g_{2}\left(2 \alpha+2 \alpha^{*}\right) W_{21}-g_{3}\left(2 \alpha^{2}+\frac{1}{2} \frac{\partial^{2}}{\partial \alpha^{* 2}}+4 \alpha \alpha^{*}+2 \alpha^{* 2}-\frac{\partial^{2}}{\partial \alpha \partial \alpha^{*}}+\frac{1}{2} \frac{\partial^{2}}{\partial \alpha^{2}}\right) W_{21} \\
& i \hbar \frac{\partial}{\partial t} W_{22}=\hbar \omega\left(\alpha^{*} \frac{\partial}{\partial \alpha^{*}}-\frac{\partial}{\partial \alpha} \alpha\right) W_{22}+g_{1}\left(\alpha+\alpha^{*}+\frac{1}{2} \frac{\partial}{\partial \alpha^{*}}-\frac{1}{2} \frac{\partial}{\partial \alpha}\right) W_{12}- \\
& -g_{1}\left(\alpha+\alpha^{*}-\frac{1}{2} \frac{\partial}{\partial \alpha^{*}}+\frac{1}{2} \frac{\partial}{\partial \alpha}\right) W_{21}-g_{2}\left(\frac{\partial}{\partial \alpha^{*}}-\frac{\partial}{\partial \alpha}\right) W_{22}-g_{3}\left(2 \alpha \frac{\partial}{\partial \alpha^{*}}-\alpha \frac{\partial}{\partial \alpha}+\frac{\partial}{\partial \alpha^{*}} \alpha^{*}+\alpha^{*} \frac{\partial}{\partial \alpha^{*}}-\frac{\partial}{\partial \alpha} \alpha-2 \alpha^{*} \frac{\partial}{\partial \alpha}\right) W_{22}
\end{aligned}
$$

The Wigner function representation (9)-(12) has the obvious advantage of simplifying the tracing out of the oscillator degrees of freedom:

$$
\operatorname{tr}_{a, a \dagger}\left[O_{\mathrm{sym}}\right]=\int d \alpha d \alpha^{*} O\left(\alpha, \alpha^{*}\right) W\left(\alpha, \alpha^{*}\right)
$$

Here "sym" indicates that in the expansion of the operator $O$ in powers of creation/annihilation operators the symmetrized operator ordering is used [1]. The diagonal terms of the density matrix in energy representation (i.e., occupation probabilities of energy levels), and therefore so called "energy entropy", can be also directly obtained from the Wigner function [10].
Let us introduce the combinations

$$
\begin{array}{ll}
\bar{W}=\frac{W_{11}+W_{22}}{2} ; & \bar{Z}=\frac{W_{11}-W_{22}}{2}, \\
\bar{X}=\frac{W_{12}+W_{21}}{2} ; & \bar{Y}=\frac{W_{12}-W_{21}}{2 i},
\end{array}
$$

which allow to expand the Wigner matrix $W_{i j}$ in Pauli matrices. One can directly express the average values of any qubit operator through these "quadratures": $\left\langle\sigma_{z}\right\rangle=\int d \alpha d \alpha^{*} Z\left(\alpha, \alpha^{*}\right)$ etc. They satisfy the equations (where $\alpha=x+i y, \alpha^{*}=x-i y$ ) 


$$
\begin{gathered}
\hbar \frac{\partial}{\partial t} \bar{W}=\hbar \omega\left(x \frac{\partial}{\partial y}-y \frac{\partial}{\partial x}\right) \bar{W}+g_{1} \frac{\partial}{\partial y} \bar{X}+\left(g_{2}+4 g_{3} x\right) \frac{\partial}{\partial y} \bar{Z} \\
\hbar \frac{\partial}{\partial t} \bar{Z}=\hbar \omega\left(x \frac{\partial}{\partial y}-y \frac{\partial}{\partial x}\right) \bar{Z}-4 g_{1} x \bar{Y}+\left(g_{2}+4 g_{3} x\right) \frac{\partial}{\partial y} \bar{W} \\
\hbar \frac{\partial}{\partial t} \bar{X}=\hbar \omega\left(x \frac{\partial}{\partial y}-y \frac{\partial}{\partial x}\right) \bar{X}+g_{1} \frac{\partial}{\partial y} \bar{W}+ \\
+\left(4 g_{2} x+8 g_{3} x^{2}\right) \bar{Y}-\frac{1}{2} g_{3} \frac{\partial^{2}}{\partial y^{2}} \bar{Y}+\hbar \Omega \bar{Y}
\end{gathered}
$$

$$
\begin{gathered}
\hbar \frac{\partial}{\partial t} \bar{Y}=\hbar \omega\left(x \frac{\partial}{\partial y}-y \frac{\partial}{\partial x}\right) \bar{Y}+4 g_{1} x \bar{Z}- \\
-\left(4 g_{2} x+8 g_{3} x^{2}\right) \bar{X}+\frac{1}{2} g_{3} \frac{\partial^{2}}{\partial y^{2}} \bar{X}-\hbar \Omega \bar{X} .
\end{gathered}
$$

In Eqs. (15)-(17) the first term (of zeroth order in qubitoscillator couplings) describes the anticlockwise rotation of the Wigner function with the oscillator frequency $\omega$. It can be removed by switching to a rotating frame,

$$
x^{\prime}=x \cos (\omega t)-y \sin (\omega t) ; y^{\prime}=x \sin (\omega t)+y \cos (\omega t) .
$$

The resulting equations are as follows:

$$
\begin{aligned}
& \hbar \frac{\partial}{\partial t} \bar{W}= g_{1}\left(-\sin (\omega t) \frac{\partial}{\partial x^{\prime}}+\cos (\omega t) \frac{\partial}{\partial y^{\prime}}\right) \bar{X}+g_{2}\left(-\sin (\omega t) \frac{\partial}{\partial x^{\prime}}+\cos (\omega t) \frac{\partial}{\partial y^{\prime}}\right) \bar{Z}+ \\
&+4 g_{3}\left(x^{\prime} \cos (\omega t)+y^{\prime} \sin (\omega t)\right)\left(-\sin (\omega t) \frac{\partial}{\partial x^{\prime}}+\cos (\omega t) \frac{\partial}{\partial y^{\prime}}\right) \bar{Z} \\
& \hbar \frac{\partial}{\partial t} \bar{Z}=-4 g_{1}\left(x^{\prime} \cos (\omega t)+y^{\prime} \sin (\omega t)\right) \bar{Y}+g_{2}\left(-\sin (\omega t) \frac{\partial}{\partial x^{\prime}}+\cos (\omega t) \frac{\partial}{\partial y^{\prime}}\right) \bar{W}+ \\
&+4 g_{3}\left(x^{\prime} \cos (\omega t)+y^{\prime} \sin (\omega t)\right)\left(-\sin (\omega t) \frac{\partial}{\partial x^{\prime}}+\cos (\omega t) \frac{\partial}{\partial y^{\prime}}\right) \bar{W} \\
& \hbar \frac{\partial}{\partial t} \bar{X}=\hbar \Omega \bar{Y}+g_{1}\left(-\sin (\omega t) \frac{\partial}{\partial x^{\prime}}+\cos (\omega t) \frac{\partial}{\partial y^{\prime}}\right) \bar{W}+4 g_{2}\left(x^{\prime} \cos (\omega t)+y^{\prime} \sin (\omega t)\right) \bar{Y}+ \\
&+8 g_{3}\left(x^{\prime 2} \cos ^{2}(\omega t)\right.\left.+y^{\prime 2} \sin ^{2}(\omega t)+2 x^{\prime} y^{\prime} \cos (\omega t) \sin (\omega t)\right) \bar{Y}-\frac{1}{2} g_{3}\left(\sin ^{2}(\omega t) \frac{\partial^{2}}{\partial x^{\prime 2}}+\cos ^{2}(\omega t) \frac{\partial^{2}}{\partial y^{\prime 2}}\right) \bar{Y} \\
& \hbar \frac{\partial}{\partial t} \bar{Y}=-\hbar \Omega \bar{X}+4 g_{1}\left(x^{\prime} \cos (\omega t)+y^{\prime} \sin (\omega t)\right) \bar{Z}-4 g_{2}\left(x^{\prime} \cos (\omega t)+y^{\prime} \sin (\omega t)\right) \bar{X}- \\
&-8 g_{3}\left(x^{\prime 2} \cos ^{2}(\omega t)+y^{\prime 2} \sin ^{2}(\omega t)+2 x^{\prime} y^{\prime} \cos (\omega t) \sin (\omega t)\right) \bar{X}+\frac{1}{2} g_{3}\left(\sin ^{2}(\omega t) \frac{\partial^{2}}{\partial x^{\prime 2}}+\cos ^{2}(\omega t) \frac{\partial^{2}}{\partial y^{\prime 2}}\right) \bar{X}
\end{aligned}
$$

These equations provide a convenient basis for numerical calculations. Note that, unlike in standard Jaynes-Cummings model ([11], Ch. 8), these equations contain counterrotating terms. Of course, a significant simplification is achieved if average over the oscillator period, $2 \pi / \omega$ :

$$
\begin{gathered}
\hbar \frac{\partial}{\partial t} \tilde{W}=2 g_{3}\left(-y^{\prime} \frac{\partial}{\partial x^{\prime}}+x^{\prime} \frac{\partial}{\partial y^{\prime}}\right) \tilde{Z} \\
\hbar \frac{\partial}{\partial t} \tilde{Z}=2 g_{3}\left(-y^{\prime} \frac{\partial}{\partial x^{\prime}}+x^{\prime} \frac{\partial}{\partial y^{\prime}}\right) \tilde{W} ; \\
\hbar \frac{\partial}{\partial t} \tilde{X}=\hbar \Omega \tilde{Y}+4 g_{3}\left(x^{\prime 2}+y^{\prime 2}\right) \tilde{Y}-\frac{g_{3}}{4}\left(\frac{\partial^{2}}{\partial x^{\prime 2}}+\frac{\partial^{2}}{\partial y^{\prime 2}}\right) \tilde{Y} \\
\hbar \frac{\partial}{\partial t} \tilde{Y}=-\hbar \Omega \tilde{X}-4 g_{3}\left(x^{\prime 2}+y^{\prime 2}\right) \tilde{X}+\frac{g_{3}}{4}\left(\frac{\partial^{2}}{\partial x^{\prime 2}}+\frac{\partial^{2}}{\partial y^{\prime 2}}\right) \tilde{X} .
\end{gathered}
$$

In this "adiabatic approximation” the diagonal and offdiagonal terms of the Wigner matrix decouple. Moreover, in the absence of nonlinear coupling, $g_{3}=0$, according to Eqs. (25), the averaged diagonal terms $\tilde{W}, \tilde{Z}$ are constant, while the off-diagonal ones, $\tilde{X}$ and $\tilde{Y}$, undergo harmonic oscillations with the qubit frequency $\Omega$. A quadratic nonlinearity causes a spread of the off-diagonal terms, while its effect on the diagonal ones reduces to a rotation of $\tilde{W}, \tilde{Z}$ (i.e., oscillations of the occupation number of the qubit's excited state) with frequency $2 g_{3} / \hbar$.

To illustrate the results, we numerically calculate the time evolution of the diagonal components of the Wigner matrix in different regimes. The initial quantum state of the system is taken as 

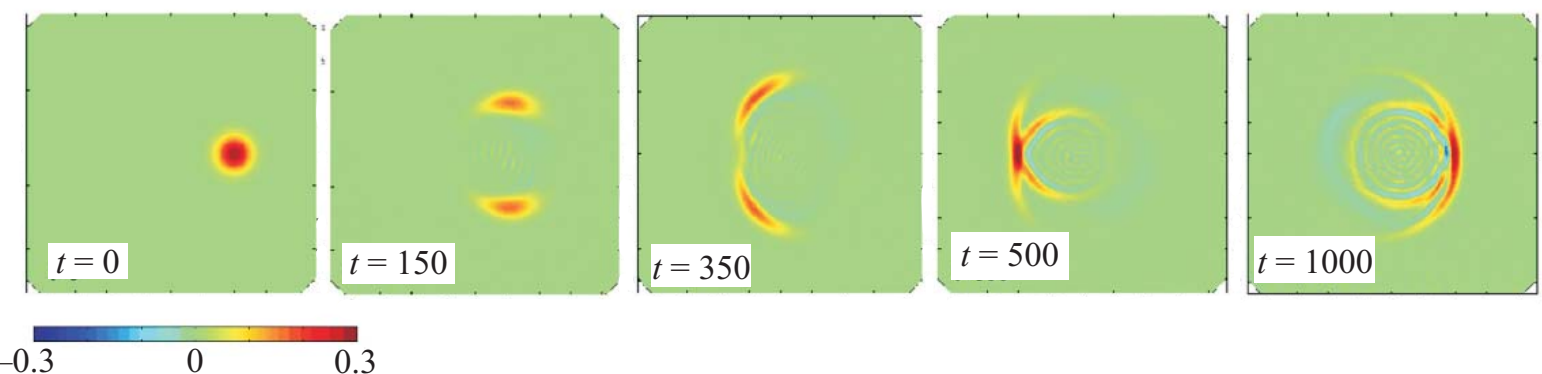

$-0.3$

0

Fig. 1. (Color online) Collapse and revival of a coherent state in an oscillator linearly coupled to a qubit. The Wigner function $\bar{W}(x, y, t)$ is plotted as a function of time. The model parameters in the Hamiltonian Eq. (1) are $\Omega=\omega=0.48 ; g_{1}=0.05 ; g_{2}=g_{3}=0$. The qubit is initially in a symmetric superposition of up and down states; the initial coherent state of the oscillator contains $\left\langle a^{\dagger} a\right\rangle=16$ photons (Eq. (25)).

$$
\left|\Psi_{0}\right\rangle=\frac{|\downarrow\rangle+|\uparrow\rangle}{\sqrt{2}} \otimes|\beta\rangle_{\text {oscillator }}
$$

where $|\beta\rangle$ is a coherent state with 16 photons.

First, consider the case of linear coupling in exact resonance ( $\left.g_{3}=0, \Omega=\omega\right)$ (Fig. 1). The periodic splitting of the initial state into two distinct states ("cat state") and its subsequent recombination is the well known phenomenon of collapse and revival, which is usually investigated using the approximate Jaynes-Cummings Hamiltonian ([11], Eq. 8.4). The results hold for a wide variety of parameters, including strong coupling $\left(g_{2} \sim \Omega, \omega\right)$, as expected, since we do not use the rotating wave approximation (Fig. 2).

(a)
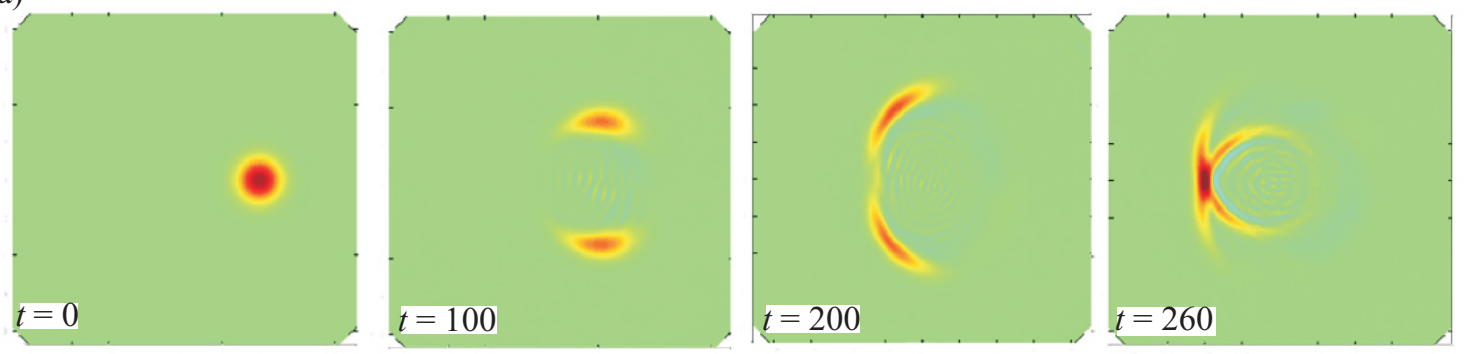

(b)
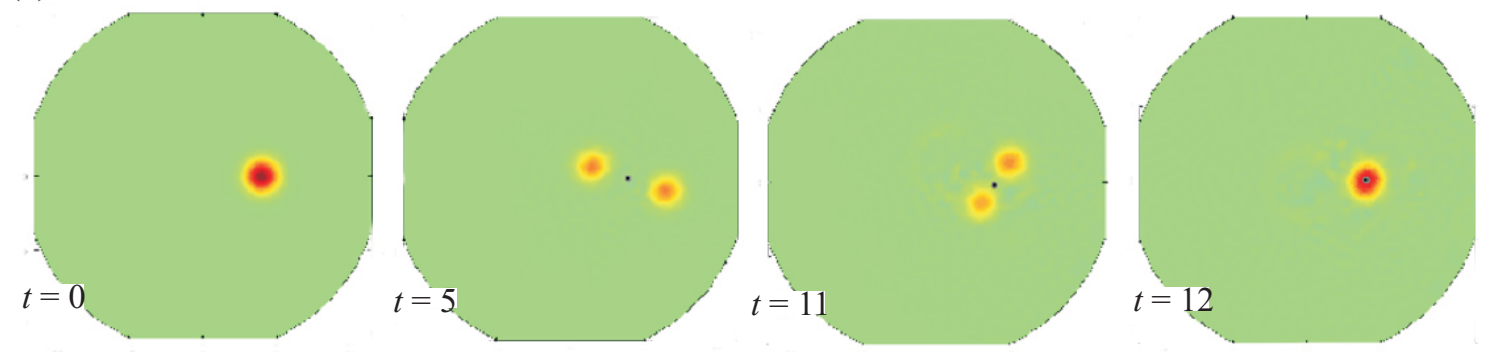

(c)
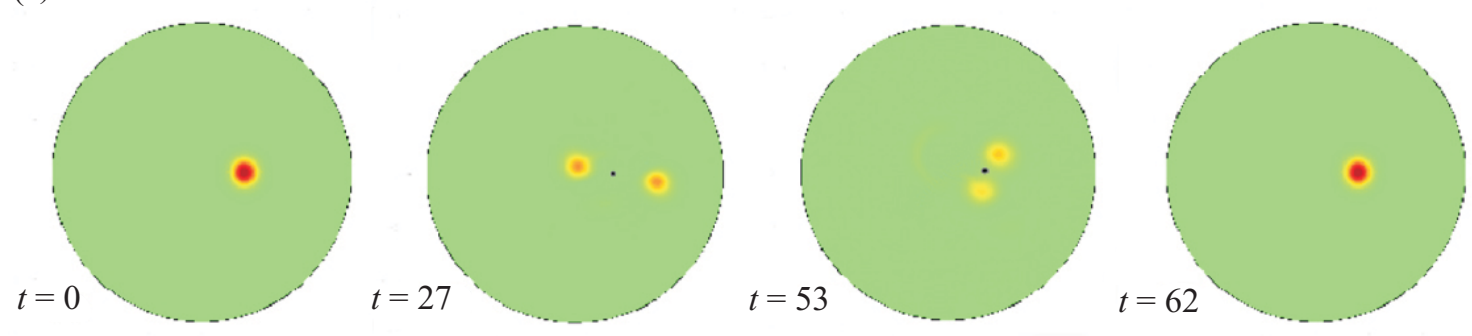

$-0.3$

0.3

Fig. 2. (Color online) Collapse and revival for varying linear coupling strength. The initial state is the same as in Fig. 1 . The Wigner function $\bar{W}(x, y, t)$ is plotted for $g_{2}=g_{3}=0$ and (a) $\Omega=\omega=1, g_{1}=0.1$; (b) $\Omega=\omega=0.5, g_{1}=0.5$; (c) $\Omega=\omega=0.1, g_{1}=0.2$. 
Introducing the non-linear (quadratic) coupling, $g_{3}$, into the numerical simulations has a drastic effect on their behaviour. Figures 3(a) and 3(b) shows the Wigner functions $\bar{W}(x, y, t)$ and $\bar{Z}(x, y, t)$ in the case of weak coupling for all three coupling terms $\left(\left(g_{1}, g_{2}, g_{3}\right)\right.$. Here, the Wigner function behaves similar to when no non-linear terms are included (Fig. 2). The collapse and revival orbits are shown with the centre at origin. However, the increase of linear coupling strength in the presence of nonlinearity causes the cat state components to move in "epicycles" as they orbit the origin (Fig. 3(c)). This causes them to miss each other, so that the revival is only partial (see Fig. 3(c) at $t=58)$. This agrees with our Eqs. (20)-(23), which show that the nonlinear term would introduce the diffusive spreading of the off-diagonal components of the Wigner function, which would then couple to the diagonal terms through the linear coupling $g_{1}$.

Numerical calculations based on the Wigner function approach are most convenient in case of large photon numbers. Nevertheless they are robust enough to be applied to small photon numbers, as illustrated in Fig. 4. Here the initial state of the qubit is still a symmetric superposition of up and down states, and the oscillator is initially in the Fock state $|2\rangle$. We consider its evolution in the presence of strong linear and weak quadratic coupling $\left(\Omega=\omega=0.1, g_{1}=g_{2}=0.1, g_{3}=0.01\right)$.

In conclusion, we have derived Bloch type equations for the motion of the Wigner function of a qubit linearly and quadratically coupled to a linear oscillator. We have done this without the use of the rotating wave approximation. We have demonstrated, using numerical simulations, the collapse and revival cycles expected for a qubit coupled to an oscillator. Our simulations appear stable over the period of one collapse and revival cycle (and longer depending upon the system parameters and the initial conditions). We have observed two clear regimes of behavior which depend on the ratio between the frequencies $(\omega, \Omega)$ and the coupling parameters. Further work could include modelling the effect dissipation and relaxation has on our system through the use of Lindblad terms in the Bloch type equations (Eqs. (20)-(23)). This will provide us with a direct and physically transparent approach to calculating the expectation values and correlations of observables in this experimentally relevant model.

We are grateful to Drs M. Everitt, S. Saveliev and R. Wilson for stimulating discussions.

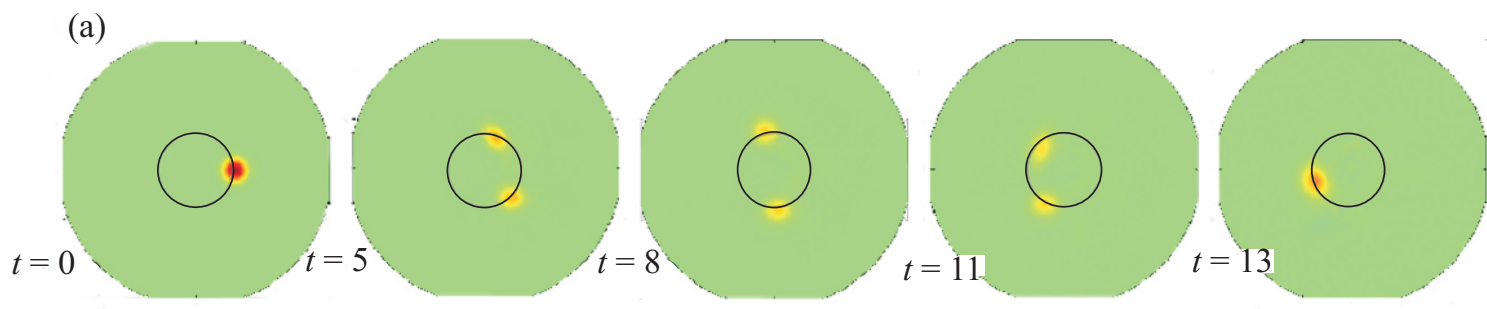

(b)
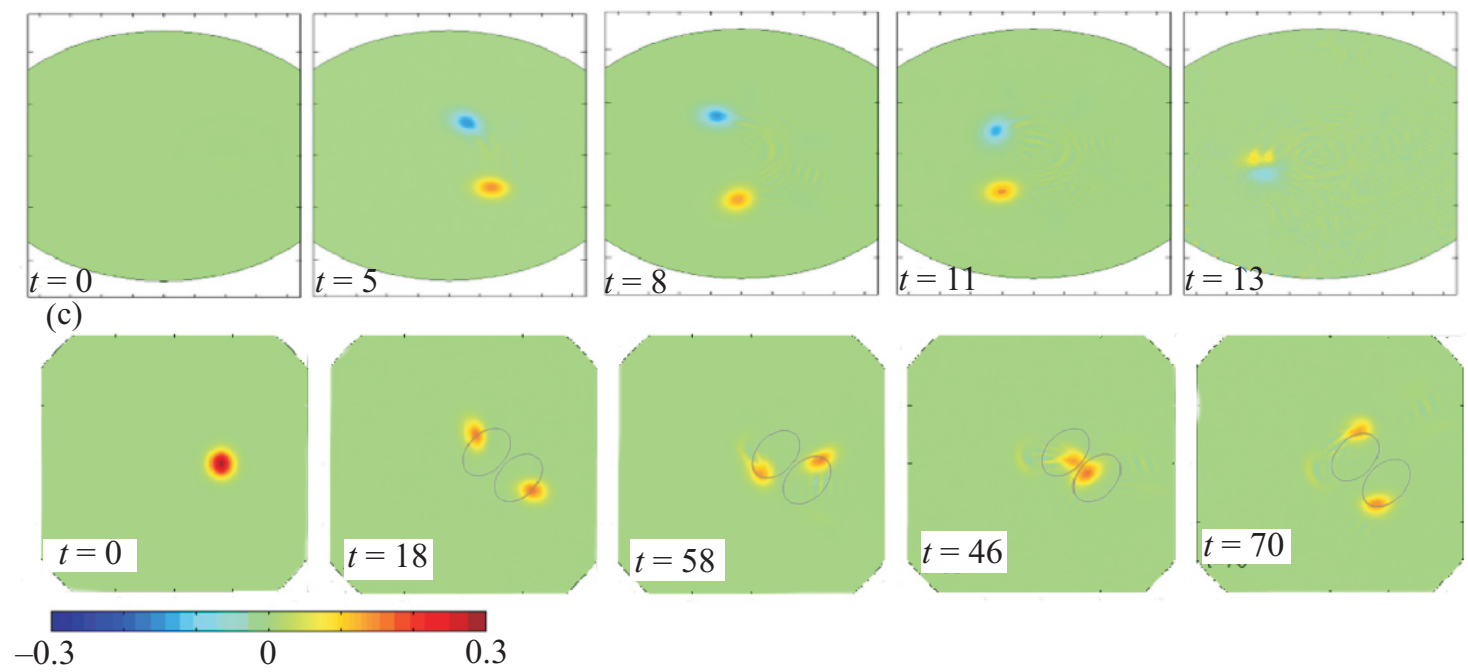

Fig. 3. (Color online) Effect of quadratic coupling on collapse and revivals of the initial state Eq. (25). In case of weak coupling $\left(\Omega=\omega=1 ; g_{1}=g_{2}=g_{3}=0.1\right)$ collapse and revival of diagonal components, $\bar{W}$ (a) and $\bar{Z}$ (b) are almost identical with the linear case ( $g_{3}=0$, Fig. 2). A relatively strong linear coupling $\left(\Omega=\omega=0.1 ; g_{1}=g_{2}=0.1 ; g_{3}=0.01\right)$ changes the trajectories of the cat state components and disrupts revivals (c). 
(a)
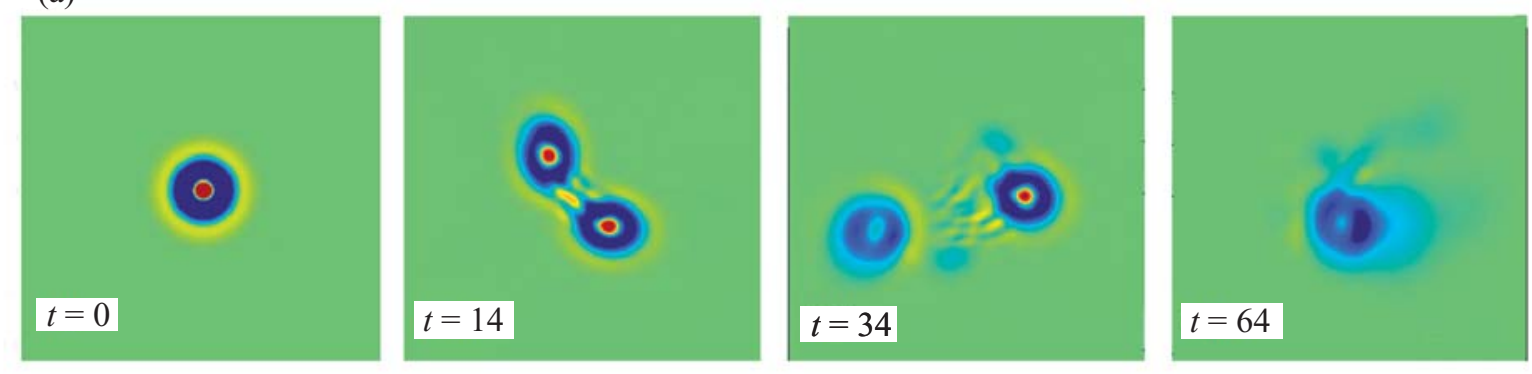

(b)
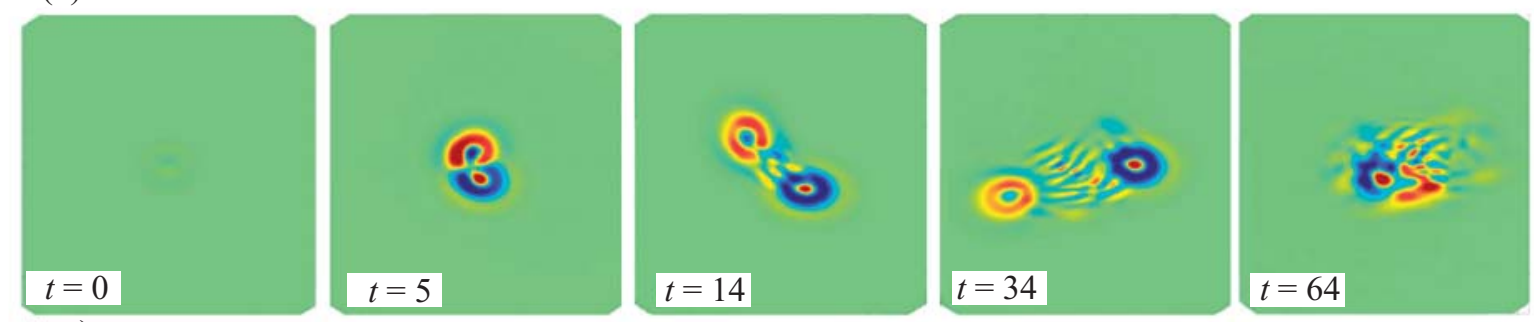

(c)
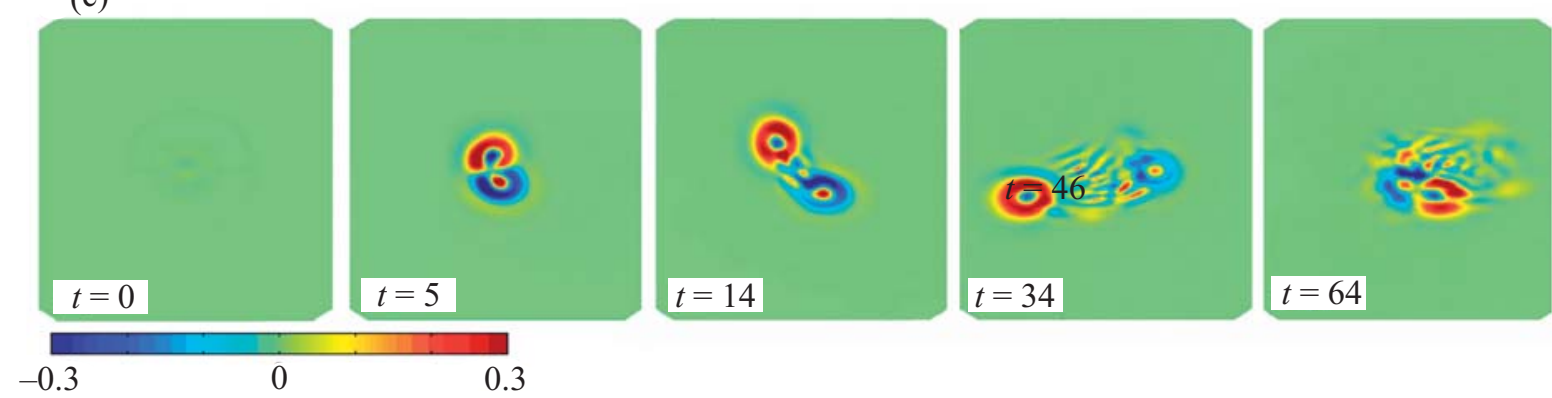

$-0.3$

0

Fig. 4. (Color online) Evolution of the initial oscillator Fock state $|2\rangle$ in the presence of strong linear and weak quadratic qubitoscillator coupling $\left(\Omega=\omega=0.1 ; g_{1}=g_{2}=0.1 ; g_{3}=0.01\right)$. The qubit is initially in a symmetric superposition of states. The components $\bar{W}, \bar{X}$ and $\bar{Z}$ are shown in panels (a), (b), and (c), respectively.

1. C.W. Gardiner and P. Zoller, Quantum Noise, Springer (2004).

2. W.P. Schleich, Quantum Optics in Phase Space, Wiley-VCH (2001).

3. A.I. Lvovsky and M.G. Raymer, Rev. Mod. Phys. 81, 299 (2009).

4. M.A. Nielsen and I.L. Chuang, Quantum Computation and Quantum Information, Cambridge University Press (2000).

5. G.D. Hutchinson, C.A. Holmes, T.M. Stace, T.P. Spiller, G.J. Milburn, S.D. Barrett, D.G. Hasko, and D.A. Williams, Phys. Rev. A 74, 062302 (2006).
6. Y. Shalibo, R. Resh, O. Fogel, D. Shwa, R. Bialczak, J.M. Martinis, and N. Katz, http://arxiv.org/abs/1208.2441.

7. W.K. Wootters, IBM J. Res. Development 48, 99 (2004).

8. D. Harland, M.J. Everitt, K. Nemoto, T. Tilmaz, and T.P. Spiller, arXiv:1210.2300 (2012).

9. A.M. Zagoskin. Quantum Engineering, Cambridge University Press (2011).

10. A.M. Zagoskin, S. Savel'ev, F. Nori, and F.V. Kusmartsev. Phys. Rev. B 86, 014501 (2012).

11. M. Orszag, Quantum Optics: Including Noise Reduction, Trapped Ions, Quantum Trajectories, and Decoherence, Springer (2007). 